Session 2793

\title{
Integration of Computational Tools in Engineering Thermodynamics Curriculum
}

\author{
Kyaw Aung \\ Department of Mechanical Engineering \\ Lamar University, Beaumont, Texas 77710
}

\begin{abstract}
Computational tools have become readily available to assist in solving many engineering problems. Thus, it is important for engineering educators to incorporate computational tools in their curriculum so that students will have first-hand knowledge and hands-on experience with these tools in their studies. This paper describes the integration of computational tools in an Engineering Thermodynamics course at Lamar University in order to emphasize the design and analysis phases of the curriculum. The computational tools employed for the course include CyclePad, a virtual laboratory software for analyzing thermodynamic power and refrigeration cycles, PsyCalc, a psychrometric calculator to determine the properties of air-vapor mixtures, and Gaseq, a chemical equilibrium program to solve equilibrium properties of gases involving chemical reaction. The reactions of students to the use of these computational tools in their coursework have been very positive. The main benefits for the students are improved understanding and insight in the design and analysis aspects of thermodynamics, and hands-on knowledge and experience of modern computational tools.

Introduction

The rapid increases in computational speed and widespread availability of computers have made possible computer-based analysis and solution of engineering problems for many industries and businesses. It is therefore imperative that engineering educators incorporate the computational tools in their curriculum so that the students will gain valuable knowledge and experience by using these tools to solve typical engineering problems. In addition, ABET has recently been emphasizing the importance of incorporating engineering design in every engineering curriculum. The integration of the computational tools in the curriculum will greatly benefit the learning process of students because these tools allow extensive use of designoriented problems such as open-ended problems and projects in the curriculum. Solving these design-oriented projects and problems provides the students with valuable insight and understanding of design and analysis processes involved in real-world engineering problems.
\end{abstract}


The main objective of this paper is to describe the integration of computational tools in the Engineering Thermodynamics II curriculum. The computational tools were integrated to emphasize the design and analysis phases of the Engineering Thermodynamics II course. The paper is based on the author's personal experiences using these tools in the Engineering Thermodynamics II class at Lamar University. The second Engineering Thermodynamic course typically covers topics including power and refrigeration cycles, properties of gas mixtures and psychrometry, second law analysis of processes and cycles, and chemical reaction processes. The computational tools employed for the course are CyclePad, a virtual laboratory software for analyzing power and refrigeration cycles, PsyCalc, a psychrometric calculator to determine the properties of air-vapor mixtures, and Gaseq, a chemical equilibrium program to solve equilibrium properties of gases involving chemical reaction. These tools are chosen because each tool features the familiar Microsoft Windows interface and costs nothing to the educators for use in the classroom environment.

The paper first describes the features, capabilities, and classroom uses of each of the computational tools. The paper next discusses the implementation of these tools and the reactions of students to the use of these computational tools. A few suggestions to improve the implementations of these tools in the thermodynamic curriculum are also provided.

Description of the Computational Tools

\section{CyclePad}

One of the most time-consuming and tedious tasks in solving thermodynamic cycles is to read the properties of the working fluid from thermodynamic tables for each state point in order to determine the desired results such as cycle efficiency and heat input. In order to evaluate the effects of a cycle parameter on the cycle performance, it is necessary to repeat the calculation of the cycle while changing the value of the parameter for each cycle. In these calculations, significant amount of time is spent in obtaining the properties for each calculation of the cycle. As a result, students spend more time getting the properties of the working fluid than analyzing the problem. Thus, it is difficult for the students to grasp the effects of the parameter on the performance of the cycle when most of the time is used for completing the computations. The CyclePad software, developed by the Qualitative Reasoning Group at Northwestern University in collaboration with the engineering faculty at Northwestern University and Oxford University ${ }^{1}$, is an ideal answer. The software uses the familiar Windows interface, and runs under Windows 95 or Windows NT. It is capable of analyzing both open and closed cycles for power and refrigeration applications. The graphical user interface (GUI) of the CyclePad software is shown in Fig. 1. When CyclePad is started, it asks the user to choose whether the problem is an open or closed cycle. Depending on the choice, the tool bar changes to display the components for an open or closed cycle. CyclePad has two modes: the Build mode allows the user to build the cycle from the components that the software provides, and the Analyze mode solves the cycle once it is completed in the build mode. In Build mode, the user specifies the working fluid and its properties at different state points of the cycle. When all specifications are completed, the user changes to the Analyze mode and the CyclePad software calculates the properties of each component in the cycle. From the properties of each component, the CyclePad software determines the cycle properties including net work done, heat input, and cycle efficiency. 


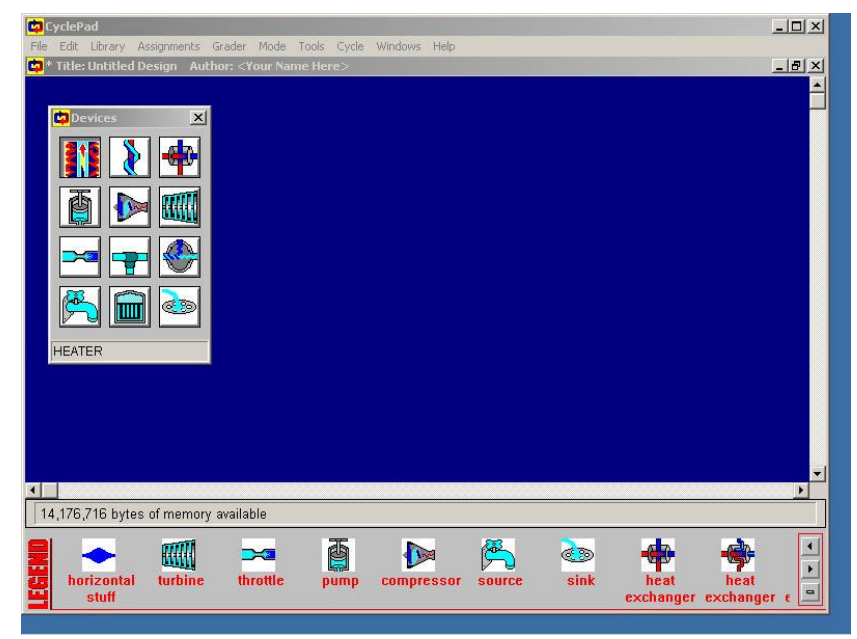

Figure 1 Graphical User Interface of CyclePad Software

The CyclePad software incorporates properties of common working fluids such as water and steam so there is no need to look up the properties from thermodynamic tables, saving a lot of time and effort for the students. As discussed earlier, using CyclePad allows students to focus more on the analysis and results rather than on doing the calculations. In addition, CyclePad provides a library containing completely solved examples of thermodynamic cycles such as vapor compression refrigeration and Rankine cycles. Thus, CyclePad makes it easier for students to understand how a particular cycle is solved, and to modify the example cycle to match the problem at hand. A typical cycle in the Build mode of CyclePad is depicted in Fig. 2.

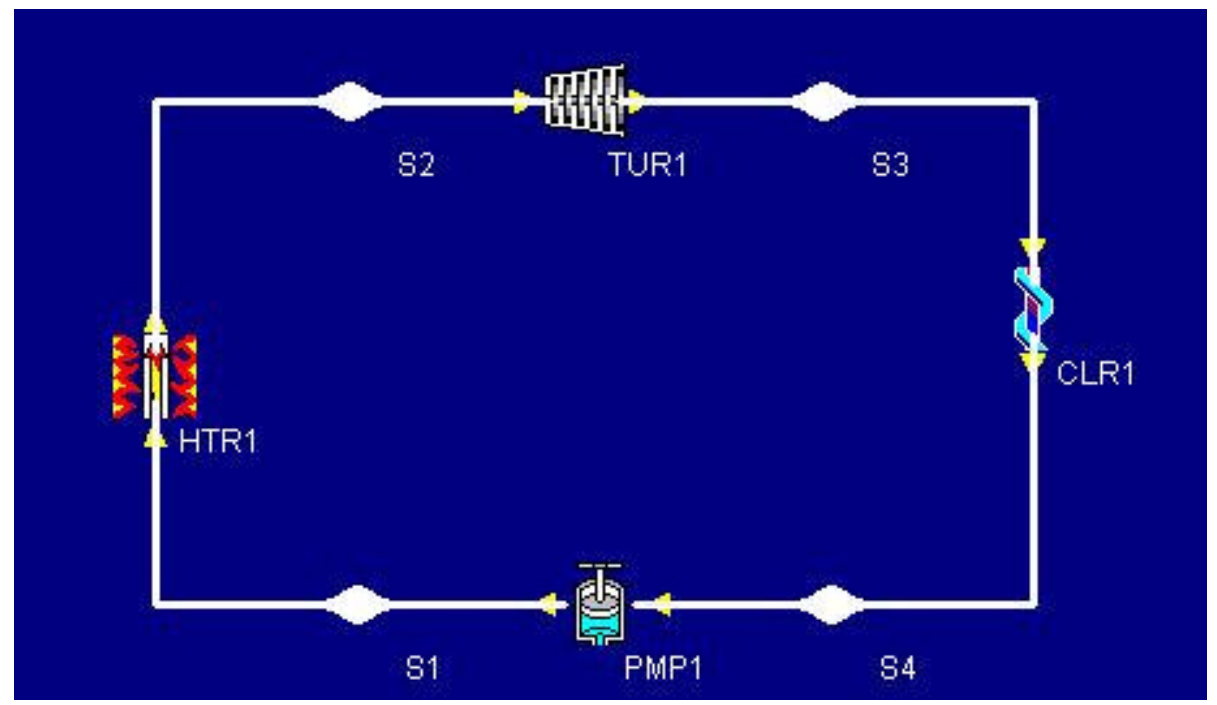

Figure 2 A Typical Cycle in CyclePad Build Mode

CyclePad provides sensitivity analysis of parameters such as pressure and temperature, and presents the results in a graphical form, making it possible to do parametric calculations on cycle parameters in a relatively short time. These features of CyclePad enable the instructor to assign students more open-ended and design problems on thermodynamic cycles. The author found the sensitivity analysis feature most useful as it allows the instructor to demonstrate the 
effects of a particular parameter to the students in class. For example, the effect of compression ratio on the thermal efficiency of an Otto cycle can be shown in a graphical form within a few minutes during class. The CyclePad software provides cost estimates for the operation of the cycle, making it useful for future implementation in the Design of Thermal Systems course at Lamar University.

To facilitate students' learning of CyclePad, the author demonstrated in class the solution of a Rankine cycle with an open and a closed feedwater heaters using CyclePad. The detailed processes of building a cycle, choosing a working fluid, assigning the properties of the working fluid at different state points, and extracting the cycle properties were demonstrated. To evaluate the effectiveness of CyclePad software, the author asked the students to solve two example problems from the textbook using both CyclePad and thermodynamic tables. The verdict of the students who carried out both analyses was unanimous: the CyclePad is incredibly useful for their understanding of thermodynamic cycles. The author assigned a design project to students during the course, asking each student to do a parametric study of cycle parameters on different cycles. With CyclePad, the students completed the design project without spending an inordinate amount of time determining thermodynamic properties. The survey of students revealed that they gained more insight into engineering thermodynamics by doing the project, and thus would like to do similar projects in other courses.

\section{PsyCalc}

In solving thermodynamic problems involving air-vapor mixtures, a psychrometric chart is typically used to determine the properties of the mixture. PsyCalc is a desktop tool developed by Linric Company to determine the psychrometric properties, and to perform mixing calculations involving air-vapor mixtures ${ }^{2}$. PsyCalc runs under Windows 3.11, Windows 95 or Windows NT, and has a familiar windows-style GUI. It provides eight psychrometric properties in both English and SI units: dry bulb temperature, wet bulb temperature, relative humidity, dew point, humidity ratio, enthalpy, vapor pressure and specific volume. A typical interface of PsyCalc for psychrometric calculations is shown in Fig. 3.

\begin{tabular}{|c|c|c|c|c|}
\hline \multicolumn{4}{|c|}{ CaSHRAE DB / MCWB Design } & $-\square x$ \\
\hline \multicolumn{5}{|c|}{ Edit Mode Help } \\
\hline \multicolumn{3}{|c|}{ Input Values... } & \multicolumn{2}{|l|}{ Select Location... } \\
\hline 由1.00 & ${ }^{*} \mathrm{~F} \mathrm{db}$ & $\bar{F}$ & USA & $\nabla$ \\
\hline 73.00 슴 & ${ }^{4} \mathrm{~F}$ wb & $\bar{z}$ & Massachusetts & $\nabla$ \\
\hline $30 \frac{1}{\sqrt{2}}$ & Alt in $\mathrm{Ft}$ & 7 & Boston & $=$ \\
\hline \multicolumn{3}{|c|}{ Output Values... } & \multirow{5}{*}{$\begin{array}{l}\text { Summer: } \\
\text { C } 0.4 \% \text { Design } \\
\text { C } 1 \% \text { Design } \\
\text { C } 2 \% \text { Design } \\
\text { winter: } \\
\text { C } 99.6 \% \text { Design } \\
\text { C9\% Design }\end{array}$} & DB / MCWB \\
\hline 36.60 & Btu/lb & $\bar{\nabla}$ & & WB / MCDB \\
\hline 14.20 & $\mathbf{f t}^{3} / \mathrm{lb}$ & 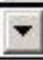 & & DP / MCDB \\
\hline 65.22 & ${ }^{*} \mathrm{~F}$ dp & \pm & & \\
\hline 93.70 & $\mathrm{gr} / \mathrm{lb}$ & 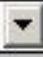 & & Do $700 \%$ \\
\hline
\end{tabular}

Figure 3 A Typical GUI Interface of PsyCalc Tool 
In addition to providing psychrometric properties, PsyCalc can perform mixing calculations between two streams of air-vapor mixtures, one of the common problems in airconditioning applications. PsyCalc also incorporates the 1997 ASHRAE climatic design data for about 1500 locations around the world, facilitating psychrometric calculations for every season at those locations. The screens of the PsyCalc tool showing ASHRAE design data and the mixing calculation are depicted in Figs. 4 and 5 respectively. In class, the author assigned homework problems involving air-vapor mixture calculations such as adiabatic saturation and evaporative cooling. The students were asked to solve the problems using both psychrometric chart and PsyCalc to compare the results and render an opinion on the use of PsyCalc. The students described the accuracy and ease of use as the main advantages in doing calculations with PsyCalc.



Figure 4 ASHRAE Weather Data in PsyCalc

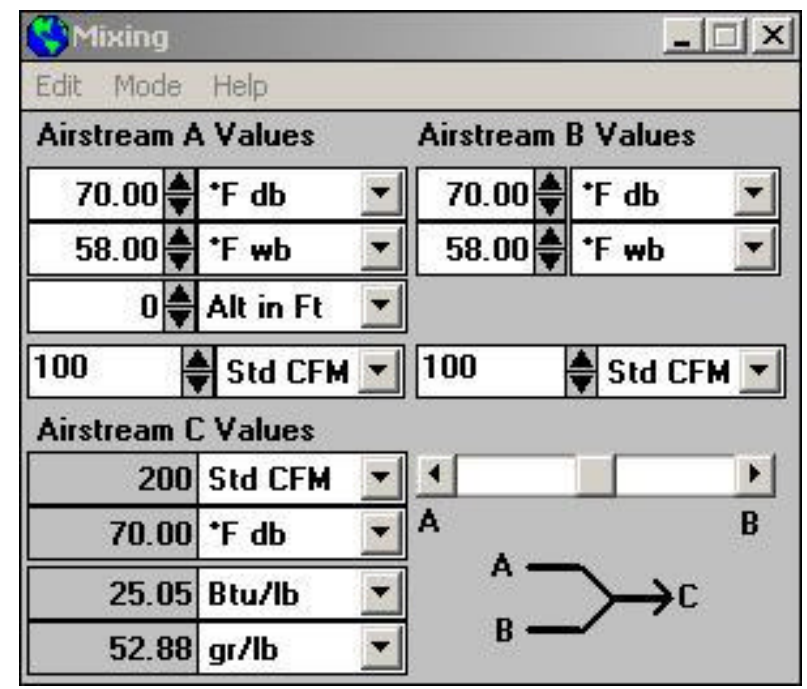

Figure 5 Mixing Calculations in PsyCalc 


\section{GasEq}

The last computational tool to be discussed is $\mathrm{Gaseq}^{3}$, a research tool developed by Christ Morley. The program features the familiar Windows interface, and runs under Windows 3.11 and Windows 95. The GUI interface of Gaseq is shown in Fig. 6.

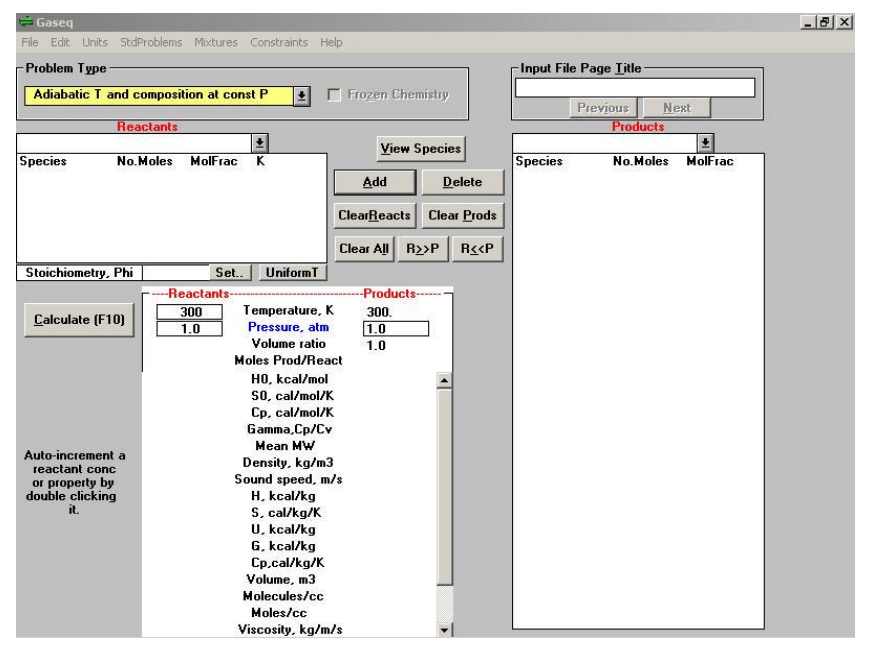

Figure 6 GUI Interface of Gaseq Program

The Gaseq program is intended for gas phase calculations involving chemical equilibrium. It can be used to determine adiabatic flame temperatures, equilibrium constants, and shock properties. Properties of common gas species such as methane, propane, oxygen, and nitrogen are built-in to the program. In addition, Gaseq can use external databases of thermodynamic properties to extend its capabilities. Gaseq can perform parametric calculations on a particular variable, and can export the results directly to the Microsoft Excel program for further analysis and plotting. An example screen showing the results from the Gaseq program is shown in Fig. 7.

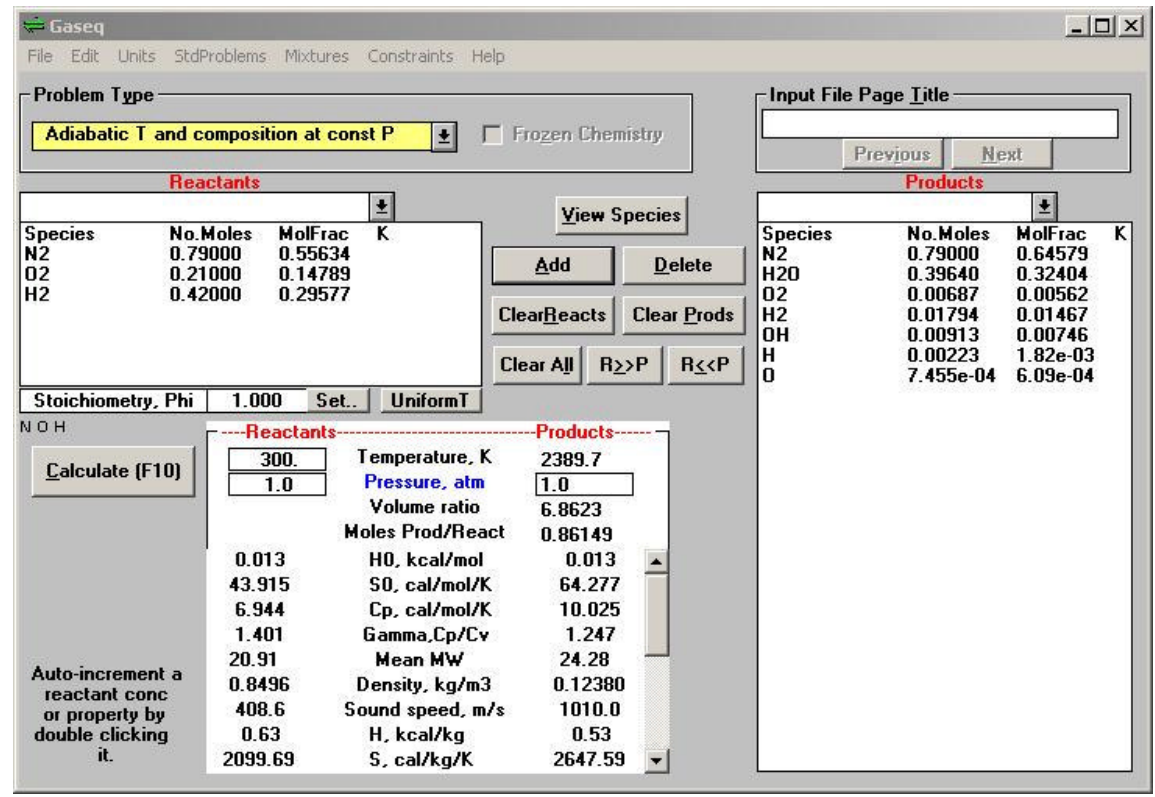

Figure 7 Calculation of Adiabatic Flame Temperature in Gaseq 
In Engineering Thermodynamic II curriculum, the last part of the course covers the chemical reactions of gas mixtures. Typical problems involving chemical reactions are the determination of the composition of combustion products and adiabatic flame temperature of different fuels. In solving these types of problems, the students need to read the properties from thermodynamic tables. An example is the calculation of adiabatic flame temperature of a stoichiometric methane/air mixture. It involves reading thermodynamic properties and calculating the flame temperature repeatedly until the converged solution is obtained. Using the Gaseq program, the students can immediately obtain the adiabatic flame temperature without referring to thermodynamic tables. Gaseq can not only save students time but also provide valuable insight into the physics of the problem. An example is the comparison of adiabatic flame temperatures of methane/air and methane/oxygen mixtures. A much higher temperature of methane/oxygen mixture compared to that of methane/air clearly shows the students the effects of nitrogen in processes involving chemical reactions.

The author generally assigns the students two problems that need to be solved by the Gaseq program: the dependence of adiabatic flame temperature of a fuel such as hydrogen on the stoichiometry, and the concentrations of $\mathrm{CO}_{2}$ and $\mathrm{CO}$ in the combustion products of methane/air flames as a function of stoichiometry. The feedback from students on the use of the Gaseq program is also positive.

Availability and implementation of the computational tools

The computational tools described in the paper were obtained directly from the web sites of each tool. The user needs to register with the developers of the CyclePad before downloading the software. For PsyCalc, the user needs to send an electronic mail directly to the Linric Company requesting a copy of the PsyCalc. The Gaseq program can be downloaded from the web site provided in the bibliography section. More information on the availability and accessibility can be obtained from the web sites listed in the bibliography section. At the time of writing this paper, all these tools are available free of charge from the developers of each tool.

Regarding implementation, each tool was made available to the students on the WebCT course web site maintained by the computer services at the Lamar University. Each student registered for the course was provided a user id and a password to access the tools. The user id and password expired at the end of the semester to prevent continued access by the students beyond the semester.

The three tools described in this paper are for use with Windows 95 and Windows NT operating systems. However, the author used these tools on computers with Windows 98 and Windows 2000 Professional operating systems without any problems.

Improvements on the use of these computational tools

The improvements on the use of these tools in the curriculum based on the experience of the author are summarized below. 
CyclePad

(1) More open-ended projects that involve designing and analyzing thermodynamic cycles rather than conducting a parametric study of the cycles

(2) Problems involving second law analysis of standard cycles using the results from the CyclePad software

PsyCalc

(1) A design project that includes various air-conditioning processes such as cooling, heating and evaporative cooling

Gaseq

(1) Problems involving parametric studies of fuel properties such as adiabatic flame temperature and $\mathrm{NO}_{\mathrm{x}}$ concentrations

(2) Problems to study the properties of different fuels at very high temperature and pressure conditions

Feedback and reactions of students

The feedback and reactions of the students on the computational tools were obtained as part of the project and problem assignments. No formal survey or questionnaire on the use of these tools was made as it was the first time these tools were implemented in the Engineering Thermodynamics II curriculum at Lamar University. The author intends to use a questionnaire on each tool to collect and analyze the responses of the students for the 2002 Fall Semester, the semester when Engineering Thermodynamics II will be offered again at Lamar University.

Conclusions

To summarize, the present paper describes the computational tools that were integrated into the Engineering Thermodynamic curriculum at Lamar University. Features, capabilities and uses of each computational tool were discussed. The main benefits for the students are improved understanding and insight into the design and analysis aspects of thermodynamics, and hands-on knowledge and experience of modern computational tools.

Bibliography

1. CyclePad, Qualitative Reasoning Group, http://www.qrg.ils.nwu.edu/projects/NSF/Cyclepad/aboutcp.htm

2. PsyCalc, Linric Inc., http://www.linric.com/psyc_1.htm

3. Gaseq, Chris Morley, http://www.c.morley.ukgateway.net/

Biography 
KYAW AUNG is an assistant professor in the Department of Mechanical Engineering at Lamar University. He received his Ph.D. degree in Aerospace Engineering from University of Michigan in 1996. He is an active member of ASEE, ASME, AIAA and Combustion Institute. He has published over 20 technical papers. 